\title{
On an asymptotic series of Ramanujan
}

\author{
Yaming Yu
}

Received: 22 May 2008 / Accepted: 1 April 2009 / Published online: 12 August 2009

(C) The Author(s) 2009. This article is published with open access at Springerlink.com

\begin{abstract}
An asymptotic series in Ramanujan's second notebook (Entry 10, Chap. 3) is concerned with the behavior of the expected value of $\phi(X)$ for large $\lambda$ where $X$ is a Poisson random variable with mean $\lambda$ and $\phi$ is a function satisfying certain growth conditions. We generalize this by studying the asymptotics of the expected value of $\phi(X)$ when the distribution of $X$ belongs to a suitable family indexed by a convolution parameter. Examples include the binomial, negative binomial, and gamma families. Some formulas associated with the negative binomial appear new.
\end{abstract}

Keywords Asymptotic expansion - Binomial distribution - Central moments · Cumulants · Gamma distribution · Negative binomial distribution · Poisson distribution · Ramanujan's notebooks

Mathematics Subject Classification (2000) Primary 34E05 · Secondary 60E05

\section{An asymptotic series of Ramanujan}

A version (modified from [2]) of Entry 10 in Chap. 3 of Ramanujan's second notebook reads

Theorem 1 Let $\phi(x), x \in[0, \infty)$, denote a function of at most polynomial growth as $x$ tends to $\infty$. Suppose there exist constants $x_{0}>0$ and $A \geq 1$, and a function $G(x)$ of at most polynomial growth as $x \rightarrow \infty$ such that for each nonnegative integer $m$ and all $x>x_{0}$, the derivatives $\phi^{(m)}(x)$ exist and satisfy

$$
\left|\frac{\phi^{(m)}(x)}{m !}\right| \leq G(x)\left(\frac{A}{x}\right)^{m} .
$$

Y. Yu (凶)

Department of Statistics, University of California, Irvine 92697, USA

e-mail: yamingy@uci.edu 
Assume that there exists a positive constant c such that

$$
G(x) \gg e^{-c \sqrt{x}}
$$

as $x \rightarrow \infty$. Put

$$
\phi_{\infty}(x)=e^{-x} \sum_{k=0}^{\infty} \frac{x^{k} \phi(k)}{k !} .
$$

Then for any fixed positive integer $M$,

$$
\phi_{\infty}(x)=\phi(x)+\sum_{n=2}^{2 M-2} \sum_{k=\lfloor(n+1) / 2\rfloor+1}^{n} b_{k n} x^{n-k+1} \frac{\phi^{(n)}(x)}{n !}+O\left(G(x) x^{-M}\right),
$$

as $x$ tends to $\infty$, where $\lfloor(n+1) / 2\rfloor$ denotes the integer part of $(n+1) / 2$ and the numbers $b_{k n}$ are defined recursively by

$$
\begin{aligned}
b_{k k} & =1, \quad k \geq 2 \\
b_{k n} & =0, \quad n<k \text { or } n>2 k-2 ; \\
b_{k+1, n+1} & =n b_{k, n-1}+(n-k+1) b_{k n}, \quad k \leq n \leq 2 k-1 .
\end{aligned}
$$

This result may seem hard to penetrate at first glance. Its relevance, however, is easily appreciated through interesting examples such as $[2,3]$

$$
e^{-x} \sum_{k=0}^{\infty} \frac{\sqrt{k} x^{k}}{k !}=\sqrt{x}\left(1-\frac{1}{8 x}-\frac{7}{128 x^{2}}+O\left(\frac{1}{x^{3}}\right)\right)
$$

and

$$
e^{-x} \sum_{k=0}^{\infty} \frac{x^{k} \log (k+1)}{k !}=\log (x)+\frac{1}{2 x}+\frac{1}{12 x^{2}}+O\left(\frac{1}{x^{3}}\right),
$$

both valid as $x \rightarrow \infty$; by choosing $\phi(x)=\log \Gamma(x+1)$ in (3), an asymptotic formula for the Shannon entropy of the Poisson distribution can also be obtained (see [6]).

The first goal of this note is a formal, probabilistic derivation of Theorem 1 . The starting point is the observation that $\phi_{\infty}(x)=E \phi(U)$, where $U$ is a Poisson random variable with mean $x$ and $E$ denotes expectation. Based on this we present in Sect. 2 a more general version (Theorem 2) of Theorem 1 by considering $U$ distributed as some distribution other than the Poisson, e.g., a gamma distribution or a binomial distribution. As illustrations, we derive asymptotic expansions for digamma functions and for inverse moments of certain positive random variables. We prove Theorem 2 in Sect 3.

Noting that $\phi_{\infty}(x)=E \phi(U)$ where $U$ has the $\operatorname{Po}(x)$ distribution, we expand $\phi(U)$ as a Taylor series

$$
\phi(U)=\phi(x)+\sum_{n=1}^{2 M-2} \frac{(U-x)^{n} \phi^{(n)}(x)}{n !}+\cdots,
$$


and formally take the expectation term by term:

$$
E \phi(U)=\phi(x)+\sum_{n=1}^{2 M-2} \frac{E(U-x)^{n} \phi^{(n)}(x)}{n !}+\cdots
$$

The quantity $\mu_{n}=E(U-x)^{n}$ is the $n$th central moment of the $\operatorname{Po}(x)$ distribution. The first few $\mu_{n}$ 's are

$$
\left(\mu_{1}, \mu_{2}, \mu_{3}, \mu_{4}\right)=\left(0, x, x, 3 x^{2}+x\right),
$$

and they obey the well-known recursion (see [19], Lemma 3, for example)

$$
\mu_{n+1}=x\left(\frac{\mathrm{d} \mu_{n}}{\mathrm{~d} x}+n \mu_{n-1}\right), \quad n \geq 2,
$$

from which we obtain, by comparing the coefficients of $x^{n-k+1}$ and using the definition of $b_{k n}$,

$$
\mu_{n}=\sum_{k=0}^{n} b_{k n} x^{n-k+1}, \quad n \geq 2 .
$$

The double sum in (3) is the result of substituting (6) in (5) and noting that $b_{k n}=0$ if $k \leq\lfloor(n+1) / 2\rfloor$. Based on this it is also clear that $\mu_{n}$ is a polynomial in $x$ of degree $\lfloor n / 2\rfloor$, which implies that, given the condition (1), the "leading term of the remainder" in (5),

$$
\frac{E(U-x)^{2 M-1} \phi^{(2 M-1)}(x)}{(2 M-1) !}=\frac{\mu_{2 M-1} \phi^{(2 M-1)}(x)}{(2 M-1) !},
$$

is $O\left(G(x) x^{-M}\right)$.

The above derivation is, of course, strictly formal. However, it can be made rigorous under the stated conditions; see Berndt [2] and Evans [5]. Berndt actually proved a modification of (3) where the order of summation over $k$ and $n$ on the right hand side is inverted and certain higher order terms of the resulting sum are absorbed in the $O\left(G(x) x^{-M}\right)$ term.

\section{A general version}

The formal derivation in Sect. 1 suggests that it is possible to generalize Theorem 1 if we let $U$ have a suitable distribution other than the Poisson. Noting the key role played by the central moments of $U$, we give a version of Theorem 1 by imposing conditions on the moment generating function (mgf) of $U$. An introduction to moment generating functions can be found in probability texts such as Gut [9]. A useful property is that, if an mgf exists in a neighborhood of zero, then for all $m \geq 1$, the $m$ th moment exists and can be obtained by differentiating the mgf $m$ times. 
Theorem 2 Let $\phi(x), x \in[0, \infty)$, denote a Borel measurable function that can be bounded in absolute value by a polynomial in $x$. Let $M$ be a fixed positive integer. Suppose there exist a constant $A \geq 1$ and a function $G(x)$ of at most polynomial growth such that for $1 \leq m \leq 2 M$ and all sufficiently large $x$, the derivatives $\phi^{(m)}(x)$ exist and satisfy

$$
\left|\frac{\phi^{(m)}(x)}{m !}\right| \leq G(x)\left(\frac{A}{x}\right)^{m} .
$$

Assume there exist $\eta \in(0,1)$ and a constant $B$ such that for all sufficiently large $x$,

$$
G(y) \leq B G(x) \text { whenever }|y-x| \leq \eta x .
$$

Let $\Omega$ be an unbounded subset of $[0, \infty)$ and let $U_{x}, x \in \Omega$, be a family of nonnegative random variables. Assume there exist a constant $\delta>0$ and a function $g(s)$ such that for all $x \in \Omega$, the mgf of $U_{x}$ exists in the interval $(-\delta, \delta)$ and satisfies

$$
E e^{s U_{x}}=e^{x g(s)}, \quad s \in(-\delta, \delta) .
$$

Assume $g^{\prime}(0)=1$ in addition. Then

$$
E \phi\left(U_{x}\right)=\phi(x)+\sum_{n=2}^{2 M-2} \sum_{k=\lfloor(n+1) / 2\rfloor+1}^{n} c_{k n} x^{n-k+1} \frac{\phi^{(n)}(x)}{n !}+O\left(G(x) x^{-M}\right),
$$

as $x$ tends to $\infty$, where $c_{k n}$ are constants that depend only on the function $g(s)$, and are determined by

$$
E\left(U_{x}-x\right)^{n}=\sum_{k=0}^{n} c_{k n} x^{n-k+1}, \quad n \geq 2
$$

Evidently, Theorem 1 is the special case $g(s)=e^{s}-1$, except for the assumption (8) on $G(x)$ which replaces (2). This new assumption does not appear very restrictive as we shall see from the examples later in this section; however it does make the proof of Theorem 2 more straightforward. We also relax the assumption on $\phi(x)$ slightly by requiring only $2 M$ derivatives.

It should be emphasized that the function $g(s)$ in (9) does not depend on $x$. Also note that $g(s)$ is analytic in $s \in(-\delta, \delta)$ given the existence of the mgf. Aside from the Poisson, examples of distribution families that satisfy (9) include the binomial, negative binomial, and gamma families. In general, suppose $Y_{1}, Y_{2}, \ldots$ is a sequence of independent and identically distributed (i.i.d.), nonnegative, nondegenerate random variables whose mgf exists in a neighborhood of zero. Then the family of random variables $\left\{\sum_{k=1}^{n} Y_{k}, n=1,2, \ldots\right\}$ ( $n$ is known as a convolution parameter) has mgf

$$
E \exp \left(s \sum_{k=1}^{n} Y_{k}\right)=\exp \left(n \log \left(E e^{s Y_{1}}\right)\right), \quad n=1,2, \ldots,
$$

which is of the form (9) with $g(s)=\left(E Y_{1}\right)^{-1} \log \left(E e^{s Y_{1}}\right)$, if we index the family by its mean $x=n E Y_{1}$. This shows that Theorem 2 is potentially applicable to a wide range of problems. 
Example 1 For a fixed $p \in(0,1)$, consider the family $U_{x}, x=p, 2 p, 3 p, \ldots$, where $U_{x}$ has the binomial distribution $\operatorname{Bi}(n, p), n=x / p$. The first few central moments of $U_{x}$ are given by $(q=1-p)$

$$
\left(\mu_{2}, \mu_{3}, \mu_{4}\right)=\left(q x,(q-p) q x, 3 q^{2} x^{2}+q(1-6 p q) x\right) .
$$

Since $\operatorname{Bi}(n, p)$ is a sum of $n$ i.i.d. $\operatorname{Bernoulli}(p)$ random variables, (9) is satisfied.

- Given $r$ (real) and $a>0$, let $\phi(x)=G(x)=(x+a)^{-r}$. It is easy to verify (7) and (8); thus we have

$$
\begin{aligned}
\sum_{k=0}^{n}\left(\begin{array}{l}
n \\
k
\end{array}\right) p^{k} q^{n-k}(k+a)^{-r}= & (n p+a)^{-r}+\frac{q r(r+1)}{2}(n p)(n p+a)^{-r-2} \\
& -\frac{(q-p) q r(r+1)(r+2)}{6}(n p)(n p+a)^{-r-3} \\
& +\frac{q^{2} r(r+1)(r+2)(r+3)}{8}(n p)^{2}(n p+a)^{-r-4} \\
& +O\left(n^{-r-3}\right),
\end{aligned}
$$

as $n \rightarrow \infty$.

- If we let $\phi(x)=x^{-r}, x \geq 1$ and $\phi(x)=0, x<1$, then we obtain an asymptotic expansion for

$$
\sum_{k=1}^{n}\left(\begin{array}{l}
n \\
k
\end{array}\right) p^{k} q^{n-k} k^{-r}
$$

by simply substituting $a=0$ in the right hand side of (11). When $r$ is a positive integer, (12) is sometimes known as the $r$ th inverse moment of the binomial. The problem of inverse moments has a long history in statistics (see, for example, Stephan [16], Grab and Savage [8], and David and Johnson [4]). More recently, expansions for (12) have been considered by Marciniak and Wesolowski [14] (see also Rempala [15]) for $r=1$, and by Žnidarič [19] for general $r$. Žnidarič [19] also gives a brief historical account with many references.

A special case corresponding to $r=-1 / 2$ is

$$
\sum_{k=0}^{n}\left(\begin{array}{l}
n \\
k
\end{array}\right) p^{k} q^{n-k} \sqrt{k}=\sqrt{n p}\left[1-\frac{q / 8}{n p}+\frac{(q-p) q / 16-15 q^{2} / 128}{(n p)^{2}}+O\left(\frac{1}{n^{3}}\right)\right],
$$

which is the binomial analog of (4) considered by Ramanujan [2].

- Let $\phi(x)=\log (x+\beta)$ for a fixed $\beta>0$ and let $G(x) \equiv 1$. We have (as $n \rightarrow \infty)$

$$
\begin{aligned}
& \sum_{k=0}^{n}\left(\begin{array}{l}
n \\
k
\end{array}\right) p^{k} q^{n-k} \log (k+\beta) \\
& \quad=\log (n p+\beta)-\frac{n p q}{2}(n p+\beta)^{-2}
\end{aligned}
$$




$$
\begin{aligned}
& +\frac{(q-p) q}{3}(n p)(n p+\beta)^{-3}-\frac{3 q^{2}}{4}(n p)^{2}(n p+\beta)^{-4} \\
& +O\left(n^{-3}\right) .
\end{aligned}
$$

The problem of approximating the left hand side of (13) appears in Krichevskiy [13] in an information theoretic context; see also Jacquet and Szpankowski [10, 11], who give an alternative derivation of (13) using the method of analytic poissonization and depoissonization. Flajolet [7] also considers similar problems using singularity analysis.

Example 2 For a fixed $p \in(0,1)$, consider the negative binomial family $\mathrm{NB}(n, p)$ whose probability mass function is $f(k ; n, p)=\left(\begin{array}{c}n+k-1 \\ k\end{array}\right) p^{n} q^{k}, k=0,1, \ldots$, where $q=1-p$. The mean is $n q / p$ and the first few central moments are

$$
\left(\mu_{2}, \mu_{3}, \mu_{4}\right)=\left(\frac{n q}{p^{2}}, \frac{n q(1+q)}{p^{3}},\left[3+\frac{6}{n}+\frac{p^{2}}{n q}\right] \frac{(n q)^{2}}{p^{4}}\right) .
$$

Similar to the binomial case, as $\mathrm{NB}(n, p)$ is a sum of $n$ i.i.d. geometric $(p)$ random variables, (9) is satisfied and Theorem 2 is applicable for an appropriate $\phi(x)$.

- Take $\phi(x)=(x+a)^{-r}, a>0$. We have, as $n \rightarrow \infty$,

$$
\begin{aligned}
\sum_{k=0}^{\infty}\left(\begin{array}{c}
n+k-1 \\
k
\end{array}\right) p^{n} q^{k}(k+a)^{-r} & \left(\frac{n q}{p}+a\right)^{-r}+\frac{r(r+1)}{2 p}\left(\frac{n q}{p}\right)\left(\frac{n q}{p}+a\right)^{-r-2} \\
& -\frac{(1+q) r(r+1)(r+2)}{6 p^{2}}\left(\frac{n q}{p}\right)\left(\frac{n q}{p}+a\right)^{-r-3} \\
& +\frac{r(r+1)(r+2)(r+3)}{8 p^{2}}\left(\frac{n q}{p}\right)^{2}\left(\frac{n q}{p}+a\right)^{-r-4} \\
& +O\left(n^{-r-3}\right) .
\end{aligned}
$$

- As in the binomial case, we obtain an asymptotic expansion for

$$
\sum_{k=1}^{\infty}\left(\begin{array}{c}
n+k-1 \\
k
\end{array}\right) p^{n} q^{k} k^{-r}
$$

for real $r$ by substituting $a=0$ in the right hand side of (14). Expansions for (15) have been considered by Marciniak and Wesolowski [14] and Rempala [15] for the special case $r=1$, and by Wuyungaowa and Wang [18] for integer $r \geq 0$.

- Let $\phi(x)=\log (x+\beta)$ for a fixed $\beta>0$. We have

$$
\sum_{k=0}^{\infty}\left(\begin{array}{c}
n+k-1 \\
k
\end{array}\right) p^{n} q^{k} \log (k+\beta)=\log \left(\frac{n q}{p}+\beta\right)-\frac{1}{2 p}\left(\frac{n q}{p}\right)\left(\frac{n q}{p}+\beta\right)^{-2}
$$




$$
\begin{aligned}
& +\frac{(1+q)}{3 p^{2}}\left(\frac{n q}{p}\right)\left(\frac{n q}{p}+\beta\right)^{-3} \\
& -\frac{3}{4 p^{2}}\left(\frac{n q}{p}\right)^{2}\left(\frac{n q}{p}+\beta\right)^{-4}+O\left(n^{-3}\right) .
\end{aligned}
$$

Example 3 Consider the gamma family $\operatorname{Gam}(x, 1), x>0$, whose density function is $f(u ; x)=u^{x-1} e^{-u} / \Gamma(x), u>0$. The mean is $x$ and the first few central moments are

$$
\left(\mu_{2}, \mu_{3}, \mu_{4}\right)=\left(x, 2 x, 3 x^{2}+6 x\right) .
$$

The moment generating function is $(1-s)^{-x}, s<1$, which is of the form (9) with $g(s)=-\log (1-s)$.

Take $\phi(x)=G(x)=x \log (x)$. We have

$$
\frac{1}{\Gamma(x)} \int_{0}^{\infty} u \log (u) u^{x-1} e^{-u} \mathrm{~d} u=x \log (x)+\frac{1}{2}-\frac{1}{12 x}+O\left(\frac{\log (x)}{x^{2}}\right),
$$

as $x \rightarrow \infty$. Noting $\Gamma(x+1)=x \Gamma(x)$, we may write

$$
\frac{\Gamma^{\prime}(x+1)}{\Gamma(x+1)}=\log (x)+\frac{1}{2 x}-\frac{1}{12 x^{2}}+O\left(\frac{\log (x)}{x^{3}}\right),
$$

which is a familiar asymptotic formula for the digamma function ([1], p. 259). By expanding for one more term we can replace $O\left(x^{-3} \log (x)\right)$ by $O\left(x^{-3}\right)$ in (16). A full asymptotic expansion can be recovered by applying (10) and using the following recursion between the central moments of $\operatorname{Gam}(x, 1)$ (see [17]):

$$
\mu_{k}=(k-1)\left(\mu_{k-1}+x \mu_{k-2}\right), \quad k \geq 2 .
$$

\section{Proof of Theorem 2}

Our proof follows Berndt [2]. In the setting of Theorem 2 we have

Lemma 1 Let $h(u), u \in[0, \infty)$, be a Borel measurable function that can be bounded in absolute value by a polynomial. Then for a fixed $t \in(0,1)$, both $E I\left(U_{x}<\right.$ tx $) h\left(U_{x}\right)$ and $E I\left(U_{x}>x / t\right) h\left(U_{x}\right)$ tend to 0 exponentially fast as $x$ tends to $\infty$, where $I(\cdot)$ is the indicator function.

Proof Observe that $x g(s)$, the cumulant generating function of $U_{x}$, is an analytic function of $s$ (real) in a neighborhood of zero. Because $g(0)=0, g^{\prime}(0)=1$ and $t \in(0,1)$, we may choose $r, \epsilon>0$ small enough such that both $g(r)<r / t$ and $g(r+$ $\epsilon)<r / t$. Since $|h(u)|$ is bounded by a polynomial, there exists a constant $D$ such that $|h(u)|<e^{\epsilon u}+D$ for all $u \in[0, \infty)$. We have

$$
\begin{aligned}
\left|E I\left(U_{x}>x / t\right) h\left(U_{x}\right)\right| & \leq E\left(e^{\epsilon U_{x}}+D\right) e^{r\left(U_{x}-x / t\right)} \\
& =e^{x[g(r+\epsilon)-r / t]}+D e^{x[g(r)-r / t]},
\end{aligned}
$$


which tends to zero exponentially as $x \rightarrow \infty$. The proof for $E I\left(U_{x}<t x\right) h\left(U_{x}\right)$ is similar and hence omitted.

Proof of Theorem 2 Throughout we assume that $x$ is sufficiently large. Define intervals $I_{1}=[0,(1-\eta) x), I_{2}=[(1-\eta) x,(1+\eta) x)$ and $I_{3}=[(1+\eta) x, \infty)$, where $\eta$ is as specified in (8).

By Lemma 1, both

$$
E I\left(U_{x} \in I_{1}\right) \phi\left(U_{x}\right)
$$

and

$$
E I\left(U_{x} \in I_{3}\right) \phi\left(U_{x}\right)
$$

tend to zero exponentially as $x \rightarrow \infty$.

Consider the Taylor polynomial

$$
\psi(y)=\sum_{k=0}^{2 M-1} \frac{\phi^{(k)}(x)}{k !}(y-x)^{k} .
$$

Since for any $y \in I_{1}$,

$$
|\psi(y)| \leq \sum_{k=0}^{2 M-1}\left|\frac{\phi^{(k)}(x)}{k !}\right| x^{k} \equiv q(x)
$$

we have

$$
\left|E I\left(U_{x} \in I_{1}\right) \psi\left(U_{x}\right)\right| \leq q(x) E I\left(U_{x} \in I_{1}\right) .
$$

From (7) it follows that $q(x)$ has at most polynomial growth as $x \rightarrow \infty$; by Lemma 1 we know that

$$
E I\left(U_{x} \in I_{1}\right) \psi\left(U_{x}\right)
$$

tends to zero exponentially as $x \rightarrow \infty$.

Similarly, for any $y \in I_{3}$, we have

$$
|\psi(y)| \leq \sum_{k=0}^{2 M-1}\left|\frac{\phi^{(k)}(x)}{k !}\right| y^{k},
$$

and hence

$$
\left|E I\left(U_{x} \in I_{3}\right) \psi\left(U_{x}\right)\right| \leq \sum_{k=0}^{2 M-1}\left|\frac{\phi^{(k)}(x)}{k !}\right| E I\left(U_{x} \in I_{3}\right) U_{x}^{k}
$$

By Lemma 1, each of $E I\left(U_{x} \in I_{3}\right) U_{x}^{k}, k \leq 2 M-1$, tends to zero exponentially as $x \rightarrow \infty$. By (7), each of $\phi^{(k)}(x)$ has at most polynomial growth as $x \rightarrow \infty$. Overall

$$
E I\left(U_{x} \in I_{3}\right) \psi\left(U_{x}\right)
$$


tends to zero exponentially as $x \rightarrow \infty$.

For any $y \in I_{2}$, there exists some point $\zeta$ between $x$ and $y$ such that

$$
\begin{aligned}
|\phi(y)-\psi(y)| & =\left|\frac{\phi^{(2 M)}(\zeta)}{(2 M) !}\right||y-x|^{2 M} \\
& \leq G(\zeta)\left(\frac{A}{(1-\eta) x}\right)^{2 M}|y-x|^{2 M} \\
& \leq B G(x)\left(\frac{A}{(1-\eta) x}\right)^{2 M}|y-x|^{2 M}
\end{aligned}
$$

where (7) and (8) are used in the inequalities. Letting $C=B[A /(1-\eta)]^{2 M}$, we have

$$
\left|E I\left(U_{x} \in I_{2}\right)\left[\phi\left(U_{x}\right)-\psi\left(U_{x}\right)\right]\right| \leq C \frac{G(x)}{x^{2 M}} E\left|U_{x}-x\right|^{2 M} .
$$

We now consider the $n$th central moment of $U_{x}, \mu_{n}=E\left(U_{x}-x\right)^{n}$, as a function of $x$. (Note that the mean of $U_{x}$ is $x$ as $E U_{x}=x g^{\prime}(0)=x$.) Expand $x g(s)$ around $s=0$ to get

$$
x g(s)=\sum_{j=1}^{\infty} \frac{x g^{(j)}(0) s^{j}}{j !}
$$

Note that the coefficient $x g^{(j)}(0)$ is the $j$ th cumulant of $U_{x}$, and, according to the well-known relation between central moments and cumulants (see [12] or [17], for example)

$$
\mu_{n}=\sum_{j=0}^{n-2}\left(\begin{array}{c}
n-1 \\
j
\end{array}\right) \mu_{j} x g^{(n-j)}(0), \quad n \geq 2,
$$

with $\mu_{0}=1$ and $\mu_{1}=0$. Based on (21), it is easy to show by induction that $\mu_{n}$ is a polynomial in $x$ of degree at most $\lfloor n / 2\rfloor$, its coefficients depending only on the function $g(s)$. Hence, for large $x$ we have $E\left(U_{x}-x\right)^{2 M}=O\left(x^{M}\right)$ and

$$
E I\left(U_{x} \in I_{2}\right)\left[\phi\left(U_{x}\right)-\psi\left(U_{x}\right)\right]=O\left(G(x) x^{-M}\right) .
$$

Combined with the exponentially small items (17), (18), (19) and (20), this gives

$$
E\left[\phi\left(U_{x}\right)-\psi\left(U_{x}\right)\right]=O\left(G(x) x^{-M}\right) .
$$

It remains to calculate $E \psi\left(U_{x}\right)$. We have, by the definition of $c_{k n}$,

$$
\begin{aligned}
E \psi\left(U_{x}\right) & =\sum_{n=0}^{2 M-1} E\left(U_{x}-x\right)^{n} \frac{\phi^{(n)}(x)}{n !} \\
& =\phi(x)+\sum_{n=2}^{2 M-1} \sum_{k=0}^{n} c_{k n} x^{n-k+1} \frac{\phi^{(n)}(x)}{n !}
\end{aligned}
$$




$$
\begin{aligned}
& =\phi(x)+\sum_{n=2}^{2 M-1} \sum_{k=\lfloor(n+1) / 2\rfloor+1}^{n} c_{k n} x^{n-k+1} \frac{\phi^{(n)}(x)}{n !} \\
& =\phi(x)+\sum_{n=2}^{2 M-2} \sum_{k=\lfloor(n+1) / 2\rfloor+1}^{n} c_{k n} x^{n-k+1} \frac{\phi^{(n)}(x)}{n !}+O\left(G(x) x^{-M}\right) .
\end{aligned}
$$

Note that the inner sum over $k$ is curtailed because the degree of $\mu_{n}$ is at most $\lfloor n / 2\rfloor$, i.e., $c_{k n}=0$ if $k \leq\lfloor(n+1) / 2\rfloor$. As a consequence of (7), the term corresponding to $n=2 M-1$ in the outer sum is written as $O\left(G(x) x^{-M}\right)$ in the last equality. The proof of (10) is now complete.

Acknowledgements The author would like to thank the reviewer for his/her valuable comments.

Open Access This article is distributed under the terms of the Creative Commons Attribution Noncommercial License which permits any noncommercial use, distribution, and reproduction in any medium, provided the original author(s) and source are credited.

\section{References}

1. Abramowitz, M., Stegun, I.A.: Handbook of Mathematical Functions. Dover, New York (1964)

2. Berndt, B.C.: Ramanujan's Notebooks, Part I. Springer, New York (1985)

3. Berndt, B.C.: Ramanujan's Notebooks, Part II. Springer, New York (1989)

4. David, F.N., Johnson, N.L.: Reciprocal Bernoulli and Poisson variables. Metron 18, $77-81$ (1956)

5. Evans, R.J.: Ramanujan's second notebook: asymptotic expansions for hypergeometric series and related functions. In: Proc. Ramanujan Centennial Conference. Academic Press, New York (1988)

6. Evans, R.J., Boersma, J., Blachman, N.M., Jagers, A.A.: The entropy of a Poisson distribution: problem 87-6. SIAM Rev. 30, 314-317 (1988)

7. Flajolet, P.: Singularity analysis and asymptotics of Bernoulli sums. Theor. Comput. Sci. 215, 371381 (1999)

8. Grab, E.L., Savage, I.R.: Tables for the expected value of $1 / x$ for positive Bernoulli and Poisson variables. J. Am. Stat. Assoc. 49, 169-177 (1954)

9. Gut, A.: Probability: A Graduate Course. Springer, New York (2005)

10. Jacquet, P., Szpankowski, W.: Analytical depoissonization and its applications. Theor. Comput. Sci. 201, 1-62 (1998)

11. Jacquet, P., Szpankowski, W.: Entropy computations via analytic depoissonization. IEEE Trans. Inf. Theory 45, 1072-1081 (1999)

12. Johnson, N.L., Kemp, A.W., Kotz, S.: Univariate Discrete Distributions, 3rd edn. Wiley, Hoboken (2005)

13. Krichevskiy, R.E.: Laplace's law of succession and universal encoding. IEEE Trans. Inf. Theory 44, 296-303 (1998)

14. Marciniak, E., Wesolowski, J.: Asymptotic Eulerian expansions for binomial and negative binomial reciprocals. Proc. Am. Math. Soc. 127, 3329-3338 (1999)

15. Rempala, G.A.: Asymptotic factorial powers expansions for binomial and negative binomial reciprocals. Proc. Am. Math. Soc. 132, 261-272 (2004)

16. Stephan, F.F.: The expected value and variance of the reciprocal and other negative powers of a positive Bernoullian variate. Ann. Math. Stat. 16, 50-61 (1945)

17. Willink, R.: Relationships between central moments and cumulants, with formulae for the central moments of gamma distributions. Commun. Stat. Theory Methods 32, 701-704 (2003)

18. Wuyungaowa and Wang, T.: Asymptotic expansions for inverse moments of binomial and negative binomial. Stat. Probab. Lett. 78, 3018-3022 (2008)

19. Žnidarič, M.: Asymptotic expansion for inverse moments of binomial and Poisson distributions. Open Stat. Probab. J. 1, 7-10 (2009) 